\title{
Stent Graft Repair of Thoracic Aortic Pathology
}

RICHARD P. CAMBRIA, M.D.

ABSTRACT Stent-graft repair of thoracic aortic pathology has progressed to phase II clinical trials of commercially made devices. The latter represent substantial improvements from previously employed homemade constructs. Preliminary experience indicates that stent-graft repair for anatomically suitable degenerative aneurysms will present an effective and less morbid approach when compared to open repair. Prospective trials of stent-graft repair for the other lesions-in-particular acute distal aortic dissections-will be required before its ultimate role is defined.

Keywords: Stent-graft, thoracic aorta

Stent graft repair of aneurysms in the thoracic aorta has progressed more slowly than the corresponding experience with infrarenal aortic lesions. The reasons for this seem obvious and are related to the much lower incidence of degenerative aneurysm in the thoracic aorta and the corresponding lack of commercial interest in development. In addition, expertise in management of thoracic aortic lesions is spread among both cardiac and vascular surgeons. However, the spectrum of thoracic aortic pathology potentially amenable to stent graft repair includes (in addition to degenerative aneurysm) patients with acute and chronic dissection, intramural hematoma, penetrating ulcer, and even traumatic aortic tear. Although the available data concerning the treatment of acute distal aortic dissection by stent graft obliteration of the

R.P.C., Professor of Surgery, Harvard Medical School, Cambridge, MA; The Division of Vascular Surgery, and the Thoracic Aortic Center, Massachusetts General Hospital, Boston, MA.

Copyright (C) 2000 by Thieme Medical Publishers, Inc., 333 Seventh Avenue, New York, NY 10001, USA. Tel.: +l(212) 584-4662. 0894-8046,p;2000,13,2,1,14,ftx,en; pvs000102 
aortic intimal tear are preliminary, it is our opinion that evolution of experience with this particular pathology will greatly increase the pool of potential candidates for stent graft repair of the thoracic aorta.

Focal pathology in the relatively straight segment of the descending aorta is perhaps the ideal circumstance to treat with stent graft repair. Alternatively, accurate proximal deployment of a stent graft in the distal aortic arch can be difficult, particularly when coupled with the force of cardiac ejection in the proximal thoracic aorta and the resultant "wind sock" effect, which is not so problematic in the abdominal aorta. The obvious parallel with abdominal aortic stent grafting is the difficult deployment in highly angulated AAA necks. Indeed, AAA neck angulation $\geq 60^{\circ}$ is an exclusionary criterion in most Food and Drug Administration (FDA)-sponsored clinical trials of AAA stent graft repair. Clearly, the normal anatomic configuration of the aortic isthmus exceeds this figure in most individuals, making stent graft deployment in this region problematic. In addition, the diameter of the distal aortic arch is sufficiently large that its length and radius of curvature are substantially larger on the "outer wall" (cephalad portion) of the distal aortic arch, compared with the corresponding dimensions on the inferior or more caudal region of the distal arch. Accordingly, thoracic stent grafts must be sized appropriately and deployed along the outer wall of the distal arch to prevent foreshortening and attachment endoleak along the inferior aspect of the aortic arch. Adjunctive surgical procedures, most commonly transposition of the left subclavian artery onto the left common carotid, may be required to permit adequate fixation length for stent grafts deployed in the transverse portion of the aortic arch (Fig. 1). Finally, coverage of intercostal vessels and the potential for spinal cord ischemic complications has been a genuine concern because the opportunity to reconstruct such vessels and/or preserve them in regions of a proximal or distal anastomosis is not possible with stent graft repair. Because $2 \mathrm{~cm}$ of fixation in relatively normal proximal and distal aorta are required for appropriate seating of currently available stent grafts, potentially more intercostal vessels are sacrificed with stent graft deployment than would occur with conventional open repair. Contemporary surgical series clearly implicate intercostal vessel sacrifice in the T9-L1 region as greatly increasing the risk of spinal cord ischemia. ${ }^{1,2}$ However, the experience to date suggests that the usual surgical paradigm with respect to intercostal vessels and the potential for paraplegia may not hold for stent graft deployment. This likely relates to factors such as aortic cross-clamp application that are obviously not used in stent graft deployment.

\section{THORACIC AORTIC ANEURYSM: NATURAL HISTORY AND CURRENT STATUS OF CONVENTIONAL REPAIR}

The general term thoracic aortic aneurysm (TAA) includes a spectrum of pathology; in addition, the natural history and surgical treatment considera- 
Fig. 1 (A) Aortogram demonstrating focal, saccular aneurysm (single arrow) originating from the inferior aspect of the distal aortic arch in a patient presenting with paralysis of the left vocal cord. Note insufficient $(<2 \mathrm{~cm})$ proximal neck (double arrow) for stent graft fixation distal to the left subclavian origin. Staged treatment with initial transposition of the left subclavian onto the left common carotid through a supraclavicular surgical approach was employed. Thereafter, stent graft deployment just distal to the left common carotid artery was carried out. (B) Intraprocedural aortogram performed after preliminary positioning of nondeployed stent graft distal to origin of left common carotid artery (arrow to left). Note filling of excluded stump of left subclavian (arrow to right) artery and filling of distal subclavian (anastomosis to common carotid not shown). (C) Initial phase of completion arteriogram demonstrating proximal stent-graft (W. L. Gore Excluder device) fixation (arrows) just beyond origin of left common carotid artery; note also absence of filling of left subclavian stump and aneurysm cavity.
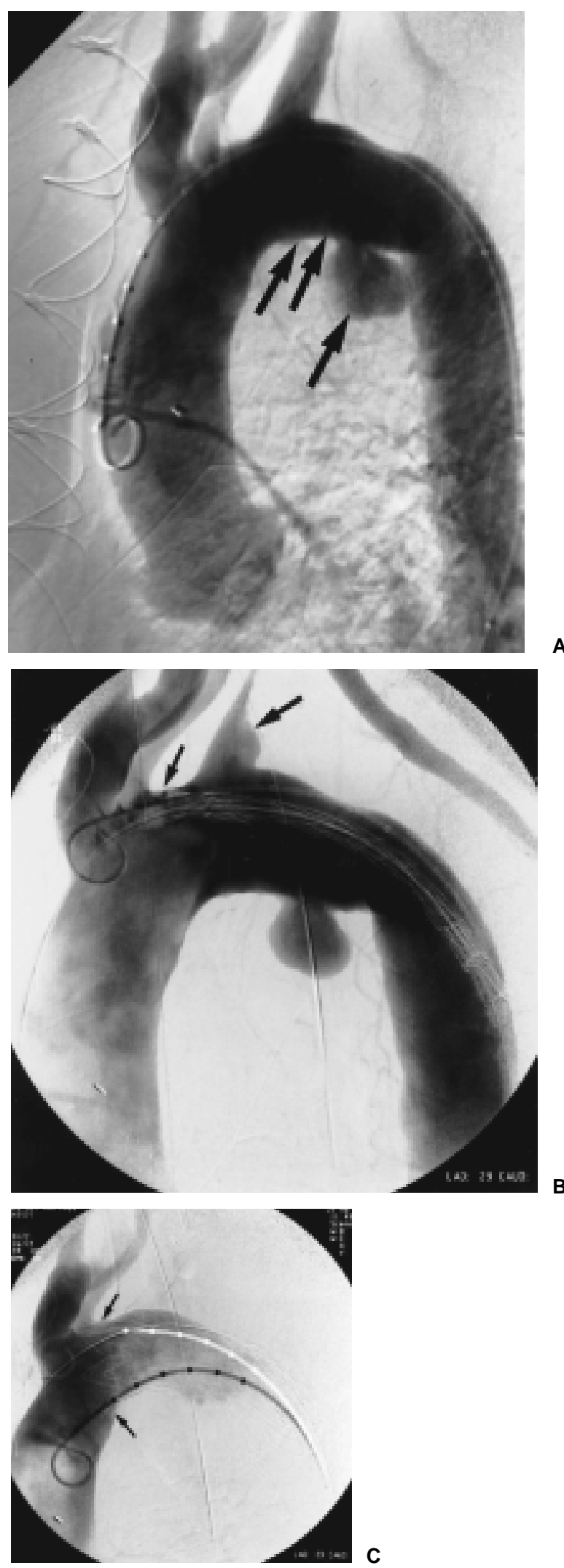
tions for ascending/arch pathology as opposed to the descending thoracic aorta may be quite different. Given the state of the art in stent graft repair in the thoracic aorta, this article will focus on lesions of the distal arch and descending aorta. The principal distinctions between TAA and AAA, of course, relate to (1) the lower incidence of TAA, (2) the corresponding dearth of natural history data for TAA, (3) the varied etiology (chronic dissection and degenerative lesions) of TAA, and (4) the acknowledged fact that the attendant morbidity and mortality of open surgical repair of TAA is expected to be significantly higher than that for AAA.

Some $80 \%$ of degenerative aortic aneurysm occurs in the infrarenal aorta, with the corresponding figure in the descending aorta being $12 \%$. Synchronous or metachronous lesions in the chest and abdomen occur in 20 to $30 \%$ of patients with degenerative aneurysms. ${ }^{3}$ Although most series of AAA report at least a $5: 1$ male to female incidence ratio, lesions involving the thoracic aorta are more common in women. In our cohort of thoracoabdominal aneurysm repairs the $\mathrm{M}: \mathrm{F}$ sex ration is $1: \mathrm{l}$; furthermore, rupture risk seems to be increased in women. ${ }^{4}$ Although the early literature mistakenly suggested that rupture risk for TAA was limited to very large $(>8 \mathrm{~cm})$ aneurysms, more recent studies have clarified this question. ${ }^{5}$ Recent natural history studies have demonstrated that rupture risk for TAA rises exponentially as the maximum diameter exceeds $6 \mathrm{~cm} .4,6$ In one study a 7-cm threshold for recommending operation was accompanied by a $23 \%$ rupture risk, clearly indicating that this threshold was overly conservative. ${ }^{7}$ In addition, this report emphasized the morbid contribution of significant chronic obstructive pulmonary disease (COPD) to increasing rupture risk, a finding reported in other natural history studies of both TAA and AAA. These data, of course, constitute a paradox in the surgical treatment of TAA — namely, significant COPD increases risk of aneurysm rupture but also contributes to respiratory morbidity accompanying open operation.

Conventional surgical treatment of TAA requires at least extensive thoracotomy, perhaps partial left heart bypass in a patient population where advanced age and comorbid conditions are commonplace. Even in contemporary reports from centers of excellence, operative mortality and spinal cord ischemic complications are in the 8 to $10 \%$ range. ${ }^{2,8}$

\section{STATE OF DEVICE EVOLUTION}

The initial thoracic aortic stent grafts were fabricated using self-expanding Gianturco Z stents that were suture attached to each other, forming an endoskeleton that was then covered by a woven Dacron graft in turn sutured to the Z-stent skeleton. This original design conceived by the Stanford group had a number of unfavorable design characteristics, including the necessity for relatively large introducer sheaths, a bulky and friction-prone construct during introduction and deployment, relative inflexibility at points of flexion and buckling in the aorta, and a deployment mechanism (graft extruded as enveloping 
introducer sheath is withdrawn) prone to "wind sock" problems with accurate deployment. ${ }^{9}$ This technical consideration has in turn led to the introduction of a number of intraprocedural maneuvers to induce temporary cardiac standstill during deployment. We have used this device in some 10 patients in a variety of difficult anatomic and clinical circumstances. Our experience mirrored that of the Stanford investigators with problems of an accurate proximal deployment and one ultimately fatal complication involving the iliofemoral access artery. Up to a third of the patients in the original Stanford series required retroperitoneal access to the abdominal aorta as a suitable access artery for stent graft introduction although this figure includes patients undergoing simultaneous open AAA repair. ${ }^{10}$ Similar to our experience with homemade endografts for AAA (using the same Dacron over Gianturco Z-stent frame), as proprietary devices become available they will obviate the need for such homemade constructs. The first of these "next generation" devices manufactured by the W. L. Gore Company is the only currently available commercial thoracic stent graft and is currently in Phase II clinical trials in 15 U.S. centers. This device is composed of an ultrathin PTFE graft supported by a continuous "exoskeleton" stent of self-expanding Nitinol. Graft compactness (and the consequent use of smaller introducer sheaths) is greatly facilitated by the ultrathin PTFE fabric and the continuous nature of the metallic stent exoskeleton (in both the longitudinal and horizontal dimensions). This allows for conformity at points of buckling and turning such as the distal arch. Most graft diameters (up to $34 \mathrm{~mm}$ ) can be delivered through a $22 \mathrm{Fr}$ introducer sheath that is positioned no higher than the abdominal aorta, from which the graft is further advanced "bare-back" over a superstiff working wire (Fig. 1B). When compared with the homemade Gianturco $\mathrm{Z}$-stent construct, this is clearly a superior device in terms of flexibility and conformity, accuracy of deployment, decreased bulkiness, and, in particular, a deployment mechanism that has largely eliminated problems of inaccurate proximal deployment. The deployment mechanism of the Excluder (W. L. Gore) device is worthy of description becauase it is both different from that employed in most stent grafts and in the circumstance of proximal thoracic stent grafts, this deployment mechanism has negated the "wind sock" effect wherein the force of ventricular ejection tends to displace the intended proximal attachment site caudally as the stent graft is extruded from its introducer sheath. The excluder device deploys by unzippering an onion-skin thin enveloping girdle, a process that occurs almost instantaneously along the length of the graft. Furthermore, the deployment is actually initiated in the graft's midregion, proceeding rapidly toward either end.

\section{PREOPERATIVE EVALUATION AND ANATOMIC SELECTION CRITERIA}

In contemporary practice we continue to utilize a combination of finecut contrast-enhanced computed tomography (CT) scanning with three- 
dimensional reconstruction and conventional arteriography performed with a graduated marker catheter for patient evaluation. These studies are complementary rather than competitive, and at this point in the evolution of thoracic aortic stent graft repair it is illogical to compromise on potentially available preoperative information. The CT scan provides the crucial information with respect to proximal and distal "neck" diameters, whereas marker catheter arteriography provides fine precision length measurements in the aortic arch, accurate estimates of overall graft length (since the catheter in situ provides an approximation of actual graft orientation as it conforms to aortic buckling), and crucial information relative to iliofemoral access arteries. Angiography also provides insight as to which oblique projections might be optimal to "open up" the aortic arch anatomy during stent graft repair. The current FDA-sponsored Phase II clinical trial of the W. L. Gore device mandates $2 \mathrm{~cm}$ of proximal and distal fixation length at either end of the TAA, with $40 \mathrm{~mm}$ being the largest graft diameter available. We typically measure aortic diameter outer wall to outer wall to allow for both the inherent wall thickness and mural atherosclerotic disease in regions of potential graft fixation. At least 10\% oversizing of endoprostheses to aortic diameter is recommended to ensure endograft sealing in the proximal and distal neck region.

Similar to the evolution of abdominal aortic stent graft repair, it is anticipated that better devices, nonprotocol use, and operator experience will increase the pool of patients treatable by endovascular repair. In a recent series utilizing first generation devices, $60 \%$ of TAA patients screened for stent graft repair were anatomically suitable for the procedure. ${ }^{11}$

\section{EXPERIENCE TO DATE}

Published data on thoracic aortic stent grafts repair are limited to first generation devices. A recently published Swedish study offers a perspective on both device and judgmental evolution. ${ }^{11}$ Twenty-five patients were treated, but a third were emergent cases, most often contained ruptures. During their experience, these investigators modified the aforementioned Dacron over Gianturco Z-stent design to include proximal fixation hooks in an effort to diminish caudal graft migration. Operative mortality was a substantial $20 \%$, and 4 of 5 deaths could be directly attributed to graft-related problems, which in turn (in this reviewer's opinion) were related to the crude constructs being used. Interestingly, massive distal embolization, which is often feared but seldom seen in our experience with abdominal aortic endografts, was responsible for two of the deaths. Careful patient selection and avoiding manipulation or attempted graft fixation in areas of atherothrombotic debris appears to be the key to eliminating this complication. Three late deaths occurred, one of which was related to procedural failure and TAA rupture. Three acute conversions were necessary, two with fatal outcomes. Lower 
extremity neurologic deficits occurred in 3 (12\%) patients, one of which was permanent paraplegia. Not surprisingly, the authors noted a significant increase in total graft length in those patients who developed spinal cord ischemic complications. Attachment site endoleak occurred in $12 \%$ of patients and late graft migration of any degree was noted in 4 of 25 patients. Patients without endoleak experienced a $13.5 \%$ decrease in TAA size at 1 year; a short proximal neck significantly predicted endoleak. This carefully documented series illustrates the evolution of judgment and technique required of those who would attempt stent graft repair of TAA. The overriding message is that problems were usually device related, and therefore overall results could be expected to improve vastly with second generation constructs.

A recent study from Austria is reminiscent of the literature on abdominal aortic stent grafting in that the authors' initial (10 patients) stent graft experience was compared with open surgical historical controls. Fifty-eight patients operated as remotely as 1989 were compared with 10 stent graft patients treated with a commercially made device. Not surprisingly, mortality (3\% vs $10 \%)$ and spinal cord injury ( $12 \%$ vs. none) rates favored the stent graft group. Primary technical success (no endoleak) was seen in 8 of 10 stent graft procedures. ${ }^{12}$

The largest experience with stent graft repair of degenerative TAA has been reported by the Stanford group. Mitchell et al. recently updated their experience with some 103 first generation implants. ${ }^{10}$ Typical of an initial experience, severe comorbidities were frequently encountered and some $60 \%$ of their patients were not suitable candidates for thoracotomy. Sixteen percent of procedures were in nonelective circumstances. Endograft configuration was a sutured/interlocking Gianturco Z-stent endoskeleton covered with woven Dacron, and due to device bulk was delivered via a 28 Fr flexible sheath. Average endograft length was $10.5 \mathrm{~cm}$, indicating a substantial percentage of patients with rather focal pathology. Abdominal aortic access was used in $30 \%$, but $2 / 3$ of these underwent conventional AAA repair at the same procedure. Follow-up was $100 \%$ complete and averaged nearly 2 years. In the analysis of their material, these authors considered all late unexplained deaths as treatment failures; complications were classified as either related or unrelated to the stent graft itself.

The Stanford group treated the spectrum of thoracic aortic pathology; $40 \%$ of their patients harbored something other than typical degenerative aneurysm. Periprocedural mortality was $9 \%$; classification as a "nonsurgical" candidate was a significant predictor of early mortality. Other significant early complications included stroke in $7 \%$ and respiratory failure in $11 \%$; however, there was but a single early conversion. Spinal cord ischemic complications were rare, with three instances of paraplegia that only occurred with concomitant or prior AAA grafting. Technical success (no endoleak) was achieved in $83 \%$ of patients. Late conversion to open repair was required in $5 \%$ of patients. Stent graft failure was associated with very proximal deploy- 
ment sites, reflecting (at least in part) the difficulty of securing first generation devices in the aortic arch. Fatal graft-related complications occurred in $4 \%$ of patients over the follow-up period. Actuarial survival at 2 years was $73 \pm 5 \%$, and due (in part) to the authors' consideration that all late unexplained deaths were considered treatment failures, only $53 \pm 10 \%$ of patients were free of treatment failure at 3.7 years.

This well-documented and honestly reported experience from the Stanford investigators permits the conclusion that device-related complications with first generation constructs are common. Arterial access complications due to large and stiff delivery systems and deployment difficulties in the aortic arch are the most prominent of these. In the near future, the second generation devices will obviate the need to consider the Dacron over Gianturco Z-stent design. In contemporary practice the latter design is still a viable option when (1) relatively focal pathology in the straight portion of the descending aorta is encountered, and (2) other device options do not exist.

\section{STENT GRAFTING FOR ACUTE AORTIC DISSECTION}

Acute aortic dissection is the most common catastrophic event affecting the aorta, with an incidence exceeding that of ruptured degenerative aneurysm. Mortality without treatment in the acute phase (within the first 2 weeks) from the central cardiaortic complications of aortic rupture and tamponade and/or aortic branch compromise is often quoted as $1 \%$ per hour over the first 72 hours. There is agreement that proximal dissections (Stanford Type A and DeBakey Types I + II) wherein the tear involves the ascending aorta require prompt surgical graft replacement to forestall rupture and tamponade. Alternatively, acute distal dissections (Stanford Type B and DeBakey Types III A + B) are typically managed with medical therapy with surgical or endovascular therapy reserved for either specific complications or failure of medical therapy. Aortic rupture is uncommon in distal dissection but does occur in $10 \%$ of cases. ${ }^{13,14}$ Typically this occurs where the aorta is largest, i.e., at the site of the intimal tear or where the dissecting process proceeds through other aneurysmal segments. Major aortic branch compromise occurs in one-third of patients and significantly increases mortality to the $50 \%$ range in our prior study of this topic. ${ }^{14}$ Not surprisingly, major branch compromise clusters among those where the dissection involves the abdominal aorta (i.e., DeBakey Types I and III B), occurring in some $50 \%$ of those so afflicted. In the majority of patients with acute proximal dissection, the immediate threat is ascending aortic rupture, and graft replacement thereof is the preferred urgent treatment. Occasionally, this threat is superseded by some peripheral branch compromise (e.g., mesenteric vascular occlusion). We and others have suggested that directed peripheral vascular intervention then becomes the treatment priority. 14,15 The bulk of early mortality in acute distal dissection is referable to aortic branch compromise. There is a growing consensus that 
specific peripheral intervention with either open surgical or endovascular means is the preferred treatment in such cases. ${ }^{14-17}$ This has largely been driven by the fact that (1) mortality with proximal descending graft repair by conventional surgical means is high and (2) depending on the mechanism of obstruction, such proximal repair may not relieve distal aortic branch obstruction.

In the chronic phase of the disease, aneurysmal degeneration of the outer wall of the false lumen may create the need for late aortic graft replacement. Although the natural history of the thoracic aorta after acute dissection has not been well studied, repetitive aortic graft replacement is common in such patients. Follow-up studies have verified that 20 to $50 \%$ of patients will require late graft replacement of (usually) thoracoabdominal aortic segments for aneurysm formation with chronic dissection as the etiology.13,18,19 These are typically complex and extensive procedures accompanied by substantial potential for surgical morbidity.

The spectrum of endovascular therapy for acute or chronic complications of aortic dissection therefore includes specific revascularization of malperfused vascular beds (usually during the acute phase of the disease) by balloon fenestration of the flap, stent deployment, or a combination thereof. These procedures are technically complex, requiring simultaneous percutaneous access to both aortic lumenae. ${ }^{16}$ The relative merits of this approach compared with open surgical methods have not been prospectively studied, and this approach is not considered further herein. The second approach is to accomplish a definitive proximal aortic stent graft repair at the site of the intimal tear, obliterating false lumen perfusion and redirecting flow into the true lumen. The goal of this strategy is to relieve aortic branch compromise, particularly when the mechanism of such relates to a bulging pressurized false lumen that greatly narrows the true lumen and compromises the branches arising from it. This so-called dynamic obstruction of aortic branches thus occurs at the aortic level with the actual branch orifice being anatomically normal. The second goal is to eliminate arterial pressure and flow into the false lumen with the expectation that this would greatly diminish the risk of late aneurysm formation.

Two recently reported clinical series detail experience with stent graft repair of acute dissection entry tears. The Stanford investigators placed first generation devices in 19 patients (4 Type A and 15 Type B) during the acute phase of the disease. ${ }^{20}$ All grafts were deployed in the descending aorta after angiographic localization of the entry tears, which were located in atypical, more distal, descending aortic locations in all but 6 patients. Obviously, the Type A patients had involvement of the ascending aorta by retrograde dissection. Anatomic requirements for stent grafting included a minimum distance of $1 \mathrm{~cm}$ between the left subclavian artery origin and the entry tear. Average entry tear length was $2 \mathrm{~cm}$ and the corresponding mean length of the stent graft was $6.9 \pm 1.5 \mathrm{~cm}$. Stent graft deployment over the entry tear was technically successful via iliofemoral access and a 22 Fr sheath in all cases; 
two patients required a second graft to completely seal the entry tear, and $80 \%$ of patients had complete thrombosis of the false lumen. Three patients (16\%) died, all with ruptured dissections or major visceral/renal ischemia. Stent graft obliteration of the entry tear was successful in reestablishing perfusion in $76 \%$ of obstructed aortic branch vessels; as expected, this was dependent on the mechanism of obstruction. So-called dynamic obstruction occurs at the aortic level and was universally remedied by stent graft obliteration of the false lumen inflow. Alternatively, in only 6 of 15 arteries wherein the dissecting process proceeded into branch vessels was complete flow restored; adjunctive uncovered stent deployment was frequently used in these vessels. The authors made no specific attempts to revascularize abdominal aortic branches whose lumenae originated from the false lumen; such branches rely on either the entry tear or other naturally occurring fenestrae for perfusion. No clinical sequelae from "ignoring" these branches were observed. With a mean follow-up duration of 13 months, no aneurysmal progression of treated aortae was observed and no aortic operations were required. Immediately after treatment, a significant reduction in diameter of the proximal descending aorta was observed.

Published simultaneously with the Stanford report, the work of Nienaber et al. considered a group of 12 patients wherein distal dissections were treated with stent grafts and compared with a historical control group of 12 patients undergoing open surgical repair for the same disease process. ${ }^{21}$ Stent graft deployment was successful in all patients with obliteration of the false lumen at 3 months after treatment and no major complications. Because the open surgical mortality was rather high at $33 \%$, the authors concluded that stent graft obliteration of the aortic entry tear is safer than conventional surgical repair.

Despite their preliminary nature, these studies offer at least encouragement, if not justification, for prospective, comparative trials of stent graft repair for acute distal dissections. The technique is both more expeditious and less complicated than endovascular fenestration and uncovered stent deployment, which has previously been used to relieve peripheral vascular manifestations of acute dissection. ${ }^{15,16}$ Furthermore, it adds the promise to impact favorably the substantial incidence of late aneurysmal dilatation of the outer wall of the false lumen and the subsequent need for aortic graft replacement.

\section{CONCLUSION}

It is perhaps premature to state that there is a controversy with respect to stent grafts in the treatment of degenerative thoracic aortic aneurysm. The available information regarding stent grafts and the clinical trials on which clinicians will make treatment decisions are just now under way. It is the 

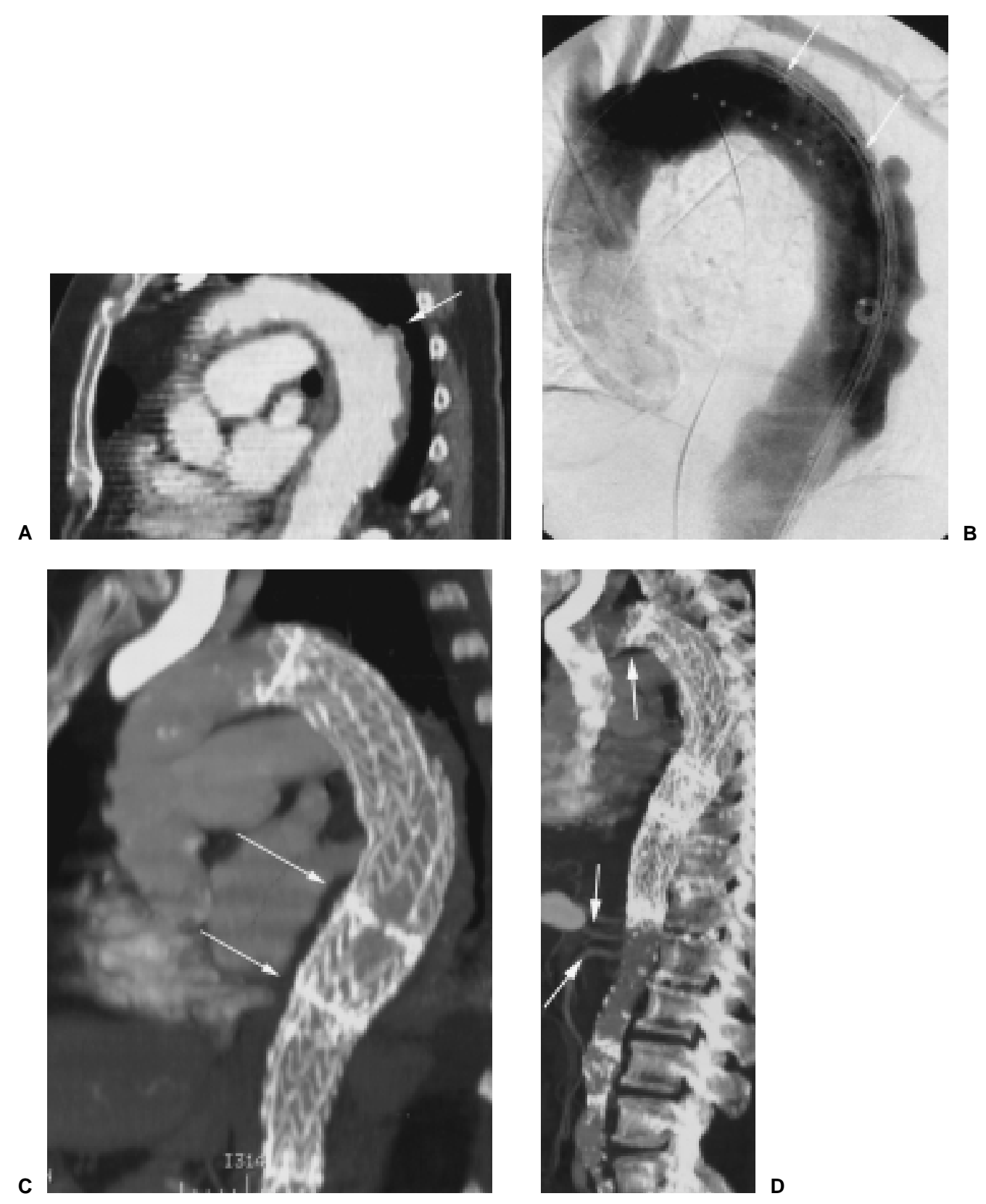

Fig. 2 Stent graft repair of a total descending aortic aneurysm in a 77-year-old female whose aneurysm had been observed to enlarge to the 7- $\mathrm{cm}$ range. (A) Preoperative 3D reconstruction of CT scan demonstrating proximal extent of TAA (arrow). (B) Intraprocedural angiogram for positioning of proximal stent-graft. Note proximal graft positioned in generous TAA neck (arrows). (C) Postoperative $3 \mathrm{D}$ reconstruction CT scan demonstrating overlap (arrows) of 2 stent-grafts in middescending aorta. (D) extent of stent graft repair from distal arch (single arrow) to just proximal to the celiac and superior mesenteric arteries (paired arrows). 
opinion of this author that stent graft repair of patients with degenerative thoracic aneurysm who would otherwise be treatable with conventional open repair should be limited to those patients participating in approved clinical trials. Early experience has been favorable, particularly considering the magnitude and potential morbidity of conventional surgical repair of extensive TAA (Fig. 2). Similarly, because the goals of stent graft repair of acute distal dissection are in substantial measure prophylactic in nature, comparative clinical trials (with presently employed medical therapy) of specific patient subsets will be needed to clarify the role of stent grafts in acute dissection.

\section{REFERENCES}

1. Safi HJ, Miller CC III, Carr C. Importance of intercostal vessel reattachment during thoracoabdominal aneurysm repair. J Vasc Surg 1998;27:58-68

2. Cambria RP, Davison JK, Carter C, et al. Epidural cooling for spinal cord protection during thoracoabdominal aneurysm repair: a five-year experience. J Vasc Surg 2000;31:1093

3. Paneton JM, Hollier LM. Basic data underlying clinical decision making. Non-dissecting thoracoabdominal aortic aneurysms. Part I. Ann Vasc Surg 1995;9:503-514

4. Clouse MD, Hallett JW, Schaff HV, et al. Improved prognosis of thoracic aortic aneurysms. JAMA 1998;280(22):1926-1929

5. McNamara JJ, Pressler VM. Natural history of arteriosclerotic thoracic aortic aneurysms. Ann Thorac Surg 1978;26:468-473

6. Perko MJ, Norgaard M, Herzog TM, et al. Unoperated aortic aneurysm. A survey of 170 patients. Ann Thorac Surg 1995;59:1204-1209

7. Juvonen T, Ergin MA, Galla JD, et al. Prospective study of the natural history of thoracic aortic aneurysms. Ann Thorac Surg 1997;63:1533-1545

8. Safi H, Campbell MP, Miller CC, et al. Cerebral spinal fluid drainage and distal aortic perfusion decrease the incidence of neurological deficit; the results of 343 descending and thoracoabdominal aortic aneurysm repairs. Eur J Vasc Endovasc Surg 1997;14:118-124

9. Dake MD, Miller DC, Semba CP, et al. Transluminal placement of endovascular stentgrafts for the treatment of descending thoracic aortic aneurysms. N Engl J Med 1994;331:1729-1734

10. Mitchell RS, Miller DC, Dake MD, et al. Thoracic aortic aneurysm repair with an endovascular stent graft: the "first generation." Ann Thorac Surg 1999;67:1971-1974

11. Greenberg RD, Resch T, Nyman U, et al. Endovascular repair of descending thoracic aortic aneurysms: an early experience with intermediate-term follow up. J Vasc Surg 2000;31:147-156

12. Ehrlich M, Grabenwoeger M, Cartes-Zumelzu F, et al. Endovascular stent graft repair for aneurysms on the descending thoracic aorta. Ann Thorac Surg 1998;66:19-24

13. DeBakeyJE, McCollum CH, Crawford ES, et al. Dissection and dissecting aneurysms of the aorta: twenty-year follow-up of five hundred twenty-seven patients treated surgically. Surgery 1982;92:1118-1134

14. Cambria RP, Brewster DC, Gertler J, et al. Vascular complications associated with spontaneous aortic dissection. J Vasc Surg 1988;7:199-209

15. Williams DM, Lee DY, Hamilton BH, et al. The dissected aorta: percutaneous treatment of ischemic complications-principles and results. J Vasc Interv Radiol 1997;8:605-625

16. Slonin SM, Nyman URO, Semba CP, et al. Aortic dissection: percutaneous management of ischemic complications with endovascular stents and balloon fenestration. J Vasc Surg 1996;23:241-253 
17. Webb TH, Williams GM. Abdominal aortic tailoring for renal, visceral and lower extremity malperfusion resulting from acute aortic dissection. J Vasc Surg 1997;26:474-481

18. Neya K, Omoto R, Kyo S. Outcome of Stanford type B acute aortic dissection. Circulation 1992;86(Suppl II):1-7

19. Schor JS, Yerlioghu ME, Galla JD, et al. Selective management of acute type B aortic dissection: long-term follow-up. Ann Thorac Surg 1996;61:1339-1341

20. Dake ME, Kato N, Mitchell RS, et al. Endovascular stent-graft placement for the treatment of acute aortic dissection. N Engl J Med 1999;340:1546-1552

21. Nienaber CA, Fattori R, Lund G, et al. Nonsurgical reconstruction of thoracic aortic dissection by stent-graft placement. N Engl J Med 1999;340:1539-1545 
Dr. Cambria has produced a thorough and concise monograph detailing the emerging technology for thoracic endografts. Although the technology is changing and the long-term durability remains unknown, we continue to share his enthusiasm for this technique in properly selected patients.

Unlike abdominal aortic aneurysms, for which modestly invasive and generally low-risk surgical repairs are well established, the repair of descending thoracic aneurysms entails significantly more mortality and morbidity, especially in the aged population. It is in this regard, specifically in their ability to reduce morbidity, that we think a second generation of endovascular devices is particularly valuable. The patients who will most benefit will undoubtedly be those with decreased cardiac, pulmonary, and renal reserve, patients for whom recovery from a conventional procedure is the most difficult. Perhaps most interesting will be the actual incidence of paraplegia and paraparesis and their relationship to previous abdominal aortic surgery and the sacrifice of low thoracic intercostal arteries. The increased maneuverability and conformability, smaller introducer size, and greater precision of deployment should minimize the complications of atheroemboli and Type I endoleaks seen with the early first generation devices.

Perhaps the most dramatic application of this technology may be for acute Type $\mathrm{B}$ aortic dissections, which are frequently managed nonoperatively and which frequently develop late complications. As Dr. Cambria has previously reported, patients presenting with acute Type B dissections and malperfusion syndromes may experience a 50 to $60 \%$ mortality even with good surgical repair. Endograft repair, by redirecting flow into the true lumen, may reverse ischemia secondary to dynamic obstruction and true lumen collapse. Malperfusion not immediately reversed by this central repair can then be immediately addressed in the cath lab by septal fenestration and ostial stenting. It is hoped that the early morbidity and mortality of this high-risk group can be averted, with an additional benefit of apparent elimination of the dual lumen aorta, which frequently undergoes aneurysmal change in the late follow-up. Restoration of a single lumen aorta in 70 to $80 \%$ of endovascular repaired patients may significantly change the natural history of Type B dissections.

Although both open repair and endograft repair for descending aneurysms are still evolving, the Phase II Food and Drug Administration (FDA) trial will compare the endovascular repair with a conventional surgical cohort. From these and subsequent trials, we can evaluate the real utility of these promising technologies.

The author is Principle Investigator for the Gore Phase II Multicenter Trial and Consultant for the W. L. Gore Company.

R.S.M., Professor, Cardiothoracic Surgery Department of Cardiovascular and Thoracic Surgery, Stanford University School of Medicine, Stanford, CA.

Copyright (C) 2000 by Thieme Medical Publishers, Inc., 333 Seventh Avenue, New York, NY 10001, USA. Tel.: +1(212) 584-4662. 0894-8046,p;2000,13,2,15,16,ftx,en; pvs00102A 
My only last word is that it is an honor to have Dr. Mitchell comment on our monograph, since he and his colleagues have been, in the opinion of this essayist, the leaders in stent graft repair of thoracic aortic pathology. Indeed much of my own chapter is merely a recapitulation of their own excellent published work. I certainly agree that extension of stent graft repair to patients with acute distal dissections is an exciting and fruitful area for further clinical research. It is our anticipation that when the current phase II trail of the W. L. Gore device is completed, that this technology will rapidly be a viable alternative for many patients with thoracic aortic disease. 
